\title{
Blockage and relative velocity Morison forces on a dynamically-responding jacket in large waves and current
}

\author{
H. Santo ${ }^{\mathrm{a}, *}$, P. H. Taylor ${ }^{\mathrm{b}}$, A. H. Day $^{\mathrm{c}}$, E. Nixon ${ }^{\mathrm{c}}$, Y. S. Choo ${ }^{\mathrm{d}}$ \\ ${ }^{a}$ Office of the Deputy President (Research and Technology), National University of Singapore, Singapore 119077, \\ Singapore \\ ${ }^{b}$ Department of Engineering Science, University of Oxford, Oxford OX1 3PJ, United Kingdom \\ ${ }^{c}$ Department of Naval Architecture, Ocean and Marine Engineering, University of Strathclyde, Glasgow G4 OLZ, \\ United Kingdom \\ ${ }^{d}$ Centre for Offshore Research $\&$ Engineering, Department of Civil and Environmental Engineering, National \\ University of Singapore, Singapore 117576, Singapore
}

\begin{abstract}
This paper documents large laboratory-scale measurements of hydrodynamic force time histories on a realistic 1:80 scale space-frame jacket structure, which is allowed to respond dynamically when exposed to combined waves and in-line current. This is a follow-on paper to Santo, Taylor, Day, Nixon and Choo (2018a) which used the same jacket structure but very stiffly supported. The aim is to investigate the validity of the Morison equation with a relative velocity formulation when applied to a complete space-frame structure, and to examine the fluid flow (and the associated hydrodynamic force) reduction relative to ambient flow due to the presence of the jacket structure as an obstacle array as well as the dynamic structural motion, interpreted as wave-current-structure blockage. Springs with different stiffness are used to allow the jacket to respond freely in the incident wavefield, with the emphasis on high frequency modes of structural vibration relative to the dominant wave frequency. Transient focussed wave groups, and embedded wave groups in a smaller regular wave background are generated in a towing tank. The jacket is towed under different speeds opposite to the wave direction to simulate wave loading with different in-line uniform currents. The measurements are compared with numerical predictions using Computational Fluid Dynamics (CFD), with the actual jacket represented in a three-dimensional numerical wave tank as a porous tower and modelled as a uniformly distributed Morison stress field derived from the relative velocity form. A time-domain ordinary differential equation solver is coupled internally with the CFD solver
\end{abstract}

\footnotetext{
*Corresponding author. Tel: +65 6516 6853. Fax: +65 67791635

Email address: dprharr@nus.edu.sg (H. Santo)
} 
to account for feedback from the structural motion into the Morison distributed stress field. An approximate expanded form of the Morison relative-velocity is also tested and is recommended for practical industrial applications. Reasonably good agreement is achieved in terms of incident surface elevation, dynamic model displacement as well as total hydrodynamic force time histories, all using a single set of Morison drag $\left(C_{d}\right)$ and inertia $\left(C_{m}\right)$ coefficients, although the numerical results tend to slightly overpredict the total forces. The good agreement between measurements and numerical predictions and the generality of the results shows that the Morison relative-velocity formulation is appropriate for a wide range of space-frame structures. In these tests, this gives rise to additional damping of the dynamic system which is equivalent to $8 \%$ of critical damping. This is significantly larger than both the structural and hydrodynamic damping combined (which is about $1 \%$ ) as quantified through free vibration (push test) in otherwise stationary water.

Keywords: Morison fluid loading, relative velocity, wave-current-structure blockage, porous block with embedded ODE simulation, spring-mass-damper system

\section{Introduction}

The standard model for the hydrodynamic force on a monopile dates back to Morison et al. (1950) who proposed a decomposition of the total force into drag and inertia components, each with an empirical coefficient to be determined from experiment. How the viscous drag and the inviscid inertia forces operate effectively independently, such that the total force can be decomposed into two distinct components, was subsequently discussed by Lighthill (1986). Since the introduction of the so-called Morison equation, it has been extensively used to characterise hydrodynamic forces on a single cylinder as well as multiple cylinders forming a space-frame offshore structure.

The applicability of the Morison equation for a statically-responding structure made of multiple cylinders, such as an offshore jacket structure, has been investigated in the past in the context of wave-current blockage. The first study dates back to Taylor (1991) who first reported the local fluid velocity and associated hydrodynamic force (mainly drag) reduction on a structure due to the presence of the structure as an obstacle array, which provides resistance to the incident wave and current flow. Therefore, the use of the Morison equation with free-stream undisturbed kinematics will tend to overpredict the actual hydrodynamic force experienced by the structure. Taylor (1991) proposed an analytical blockage model accounting for blockage effect due to steady current flow 
(hence the term 'current blockage') as an improvement to the Morison equation for multi component space-frame structures. Subsequently, Taylor et al. (2013) and Santo et al. (2014b) extended the analytical work to include regular waves with in-line current, and demonstrated the additional force reduction due to the extra contribution from waves into the hydrodynamic loading and this was coined as 'wave-current blockage'. Their results have been extensively validated by experiments from small lab-scale tests involving perforated flat plates to large-scale tests using a scaled jacket model in a large towing tank, as well as numerical CFD simulations using a porous block as a proxy for such structural models, see Santo et al. (2014a,b, 2015, 2017, 2018a). It is worth stressing that a universal form of reduction factors to reduce the undisturbed flow kinematics to account for wavecurrent blockage, similar to reduction factors for current blockage given in the design standard API RP 2A (2000), cannot be obtained. Therefore, it is necessary to solve for the blocked (or disturbed) kinematics accounting for the presence of the structure using numerical CFD simulations.

For dynamically-responding structure consisting of multiple cylinders, an extension of the Morison equation, usually termed the Morison with relative-velocity formulation, includes interaction terms that involve both fluid and structural velocities and accelerations. The validity of the Morison relative-velocity form has been investigated in the past, although most of the studies primarily considered a single cylinder oscillated in otherwise still water, see for example Moe and Verley (1980); Williamson (1985); Shafiee-Far et al. (1996); Burrows et al. (1997); Sumer and Fredsøe (2006). It is thus of interest to investigate this experimentally using a full jacket model allowed to respond dynamically when subjected to a range of waves with in-line current.

This paper serves as an extension to previous research on wave-current blockage on staticallyresponding (fixed bottom-founded) space-frame offshore structures. Here we attempt to address the following two questions: is the relative velocity version of the Morison equation adequate when applied to a dynamically-responding space-frame structure? If so, can the problem be treated in a similar way as the blockage effect on a statically-responding structure, with some modification to the underlying Morison form? Examples of dynamically-responding structures in offshore engineering include compliant towers, jack-ups and deepwater jackets. The aim of this paper is to investigate whether the relative structural motion also contributes to any additional blockage effects, which then provide additional fluid damping and cause the net dynamic motion of the structure to be (much) smaller. We will describe this as 'wave-current-structure blockage'.

Indeed there have been some evidence from the existing compliant towers reported by the 
industry. For example, full-scale field measurements on the Exxon Lena guyed compliant tower, as reported by Steele (1986), concluded that the response of the tower to Loop Current eddies was overpredicted by a factor of five to six. Thus, the net current velocity within the tower must have been only $40 \%$ of the far field velocity, a significant $60 \%$ flow reduction!

Previous work by Santo et al. (2018c) and Santo et al. (2018b), who looked at forced oscillations on grids of perforated plates, provides an indication that Morison relative-velocity formulation can adequately describe the complete measured force-time history after accounting for wave-currentstructure blockage. Although the small-scale experiments were conducted using idealised geometry representing a space-frame structure at low Reynolds number, that study provides the motivation for the present work to conduct a more realistic series of experiments at larger scale. In particular, this paper focusses on a flow regime where the frequency of the primary structural resonance is higher than the dominant wave frequency, thus representing either the second mode of a compliant tower or the first mode of jack-up legs. The structural velocity in this flow regime is typically smaller than the undisturbed wave kinematics, at least close to the free-surface.

The paper is arranged as follows. The definition of blockage will be further clarified in the next section in view of potential confusion with 'wind tunnel blockage' and 'wave-current blocking'. The experimental modelling and data analysis will be described in Section 3, followed by the numerical modelling and results in Section 4. The same section also contains an exploration of a simplified version of the relative velocity formulation suitable for straightforward adoption by industry. Section 5 contain some discussions such as additional damping and the scaling effects, while Section 5 concludes the paper.

\section{Definition}

Our use of the term wave current structure blockage is consistent with our previous work, starting with Taylor (1991). However, we stress that it is not directly related to two other uses of the term 'blockage' in the fluid dynamics literature. We define current blockage as the effect of flow resistance due to an array of closely spaced obstacles on the total force on the array. Upstream, the approaching flow diverges away from the array because of the resistance within the array. Downstream, the individual wakes of the obstacles merge and the local pressure rises back to ambient as the bulk wake expands. The net effect of both the upstream irrotational divergence and the downstream rotational wake relaxation is to reduce the average velocity within the obstacle 
array to less than the speed of the approach flow far upstream. Other than by the ocean freesurface, there is no other lateral constraint on the flow. This is simple current blockage and in our more recent publications, we have explored the effects of a large in-line oscillation, to represent wave kinematics superimposed on an ocean current, hence wave-current blockage. In this paper, the structure is allowed to respond dynamically under the fluid loading, hence wave-current-structure blockage.

In contrast, wind tunnel blockage refers to the effects of wind tunnel internal surfaces on the performance of a model within, be this lift on a wing or drag on a bluff body. Any body within the flow causes a disturbance to the incident flow and this flow will diverge outwards away from the body. The bounding tunnel walls, floor and roof provide fixed boundaries that the flow cannot pass through. Hence, there is an effect on the local flow at the body surface, both velocity and the pressure, resulting from the finite size of the wind tunnel. The forces exerted on the body are then different to what they would have been if the body had been in an effectively infinite flow. Thus, wind tunnel blockage is a consequence of both changes to the essential inviscid bulk surrounding flow and the local rotational wake. Early work on wind tunnel blockage can be found in Glauert (1933) and Maskell (1963).

A second use of the term wave-current blocking refers to the inability of a wave to propagate upstream against a fast flow, the current. This can be an entirely inviscid phenomenon. See the extensive presentations by Peregrine (1976) and Jonsson (1990), and the recent paper by Das et al. (2018). In our study, the characteristic speeds for free-surface waves, the group velocity and the phase speed, are both much larger than the ocean current. Also, we have the wave and the current inline through the space-frame, so the instantaneous fluid kinematics are maximized. Then, the flow is far from any regime where wave-current blocking can occur.

\section{Experimental modelling and data analysis}

\subsection{Experimental setup}

These experiments were conducted in the towing tank of the Kelvin Hydrodynamics Laboratory, University of Strathclyde, Glasgow. The tank is $76 \mathrm{~m}$ long, $4.6 \mathrm{~m}$ wide and $2.5 \mathrm{~m}$ deep. Four paddles of Edinburgh Design Limited (EDL) 'flap-type' wavemakers with force-feedback are located at one end, while a sloping beach acting as a passive absorber is placed at the other end. In the experiments, 

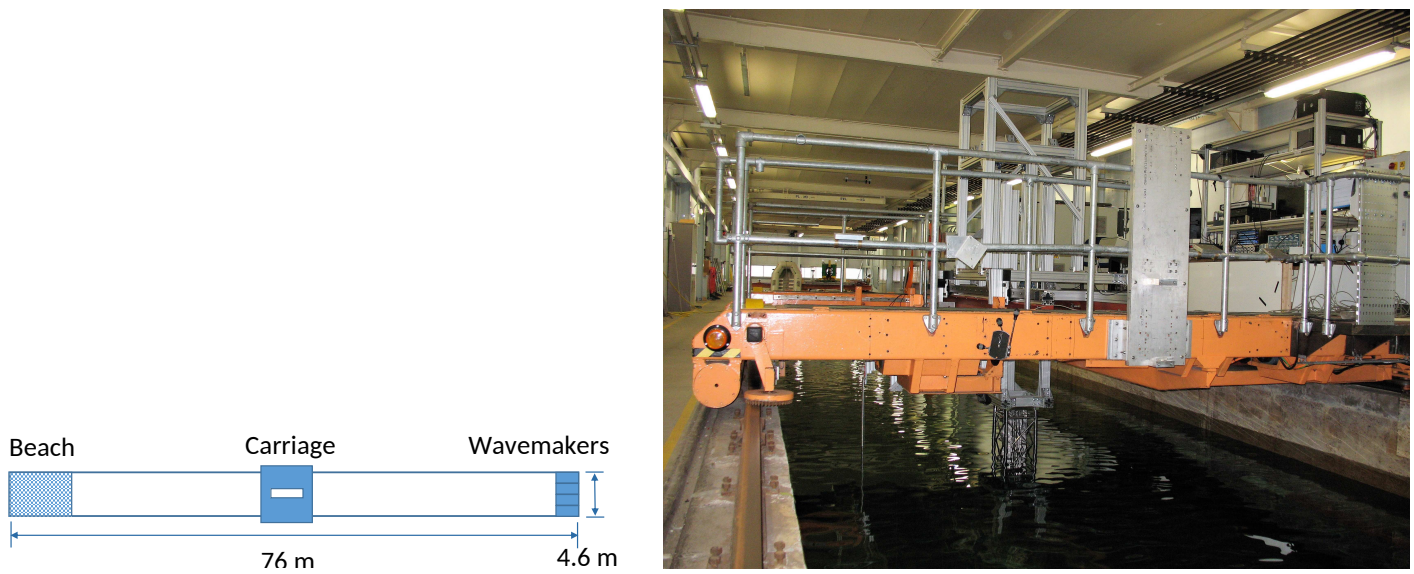

Figure 1: Left panel shows the plan view of the towing tank facility (not to scale). Right panel shows a photograph of the carriage when viewed in a downstream direction along the tank. On the carriage, a parallel pendulum system supports the jacket model below. Also shown is a wave gauge next to the jacket model.

linear wave generation was used. A self-propelled carriage runs along the longitudinal direction of the tank. Figure 1 shows a plan view as well as a photographs of the carriage.

We use the same 1:80 jacket model as used previously in Santo et al. (2018a). The jacket model was hung below the carriage, which was towed at constant speed along the tank to simulate uniform current. Figure 2 shows a 3D CAD model of the jacket with relevant geometric information (left) and a plan view of the jacket (right). In these experiments, only the end-on configuration was tested, as this will provide more blockage and a more severe test of the modelling.

The jacket model was suspended by a mounting frame with a parallel pendulum arrangement (or inverted table), such that the still water level is at $0.12 \mathrm{~m}$ below the centre of the top X-brace, or a distance of $1.33 \mathrm{~m}$ up from the jacket base. The mounting frame was then hung below a rigid frame attached on the towing carriage, see Figure 3. Such arrangement allows the total horizontal hydrodynamic load to be measured directly by a force transducer. The force transducer was rated at $50 \mathrm{~kg}(490 \mathrm{~N})$ and sampled at $5555.6 \mathrm{~Hz}$. A resistance-based wave probe, sampled at the same rate as the force transducer, was mounted on the towing carriage midway between the jacket model and the side of the tank to provide phase information of the incident waves. A Qualysis motion tracking system is used to record the displacement of the jacket model during dynamic tests.

Previously in Santo et al. (2018a), the mounting frame was connected directly to a force transducer via a rigid link in order to measure the total horizontal hydrodynamic load during static 

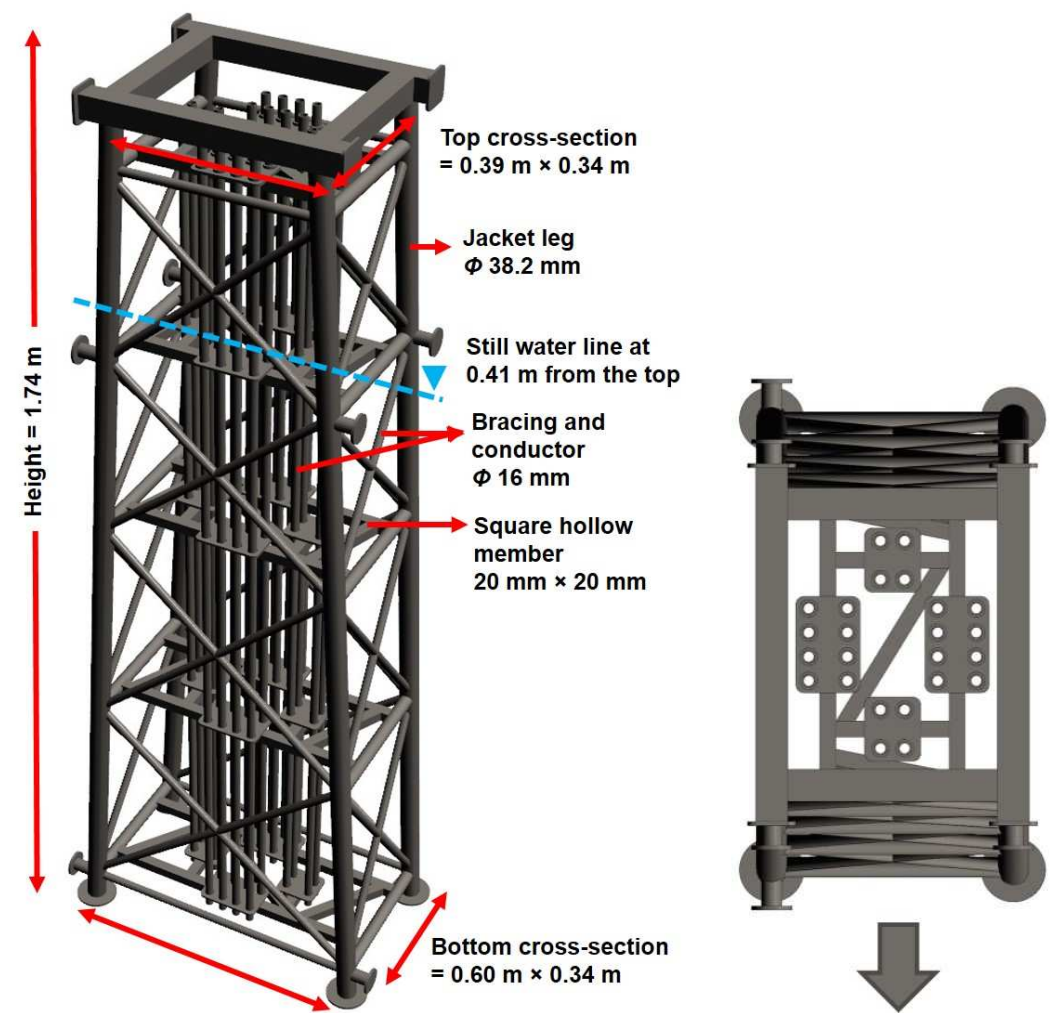

Figure 2: Left picture shows a 3D CAD model of the jacket with relevant geometric information. Right picture shows the plan view of the jacket.

(very stiff) tests. In these new dynamic tests, one spring was attached in between the mounting frame and a force transducer at the front of the setup, and another identical spring in between the mounting frame and a rigid frame at the rear of the setup (without any force transducer), as shown in Figure 3. The springs were pre-tensioned equally at each side at adequate extension to prevent them from going completely slack or the spring helix making self-contact. This minimises any nonlinearity in the behaviour of the spring at any point in the motion. It is worth remarking that since the force measurement is only taken at one side of springs during the tests, the analysis inevitably requires us to assume that both sides of springs are equally stiff and therefore identical.

Two sets of springs with two different total stiffness $(4600 \mathrm{~N} / \mathrm{m}$ and $8280 \mathrm{~N} / \mathrm{m})$ were used in parallel sets of experimental tests, hereafter denoted as spring 1 and spring 2 arrangement, respectively. These are chosen to yield high frequency modes of structural vibration relative to the 

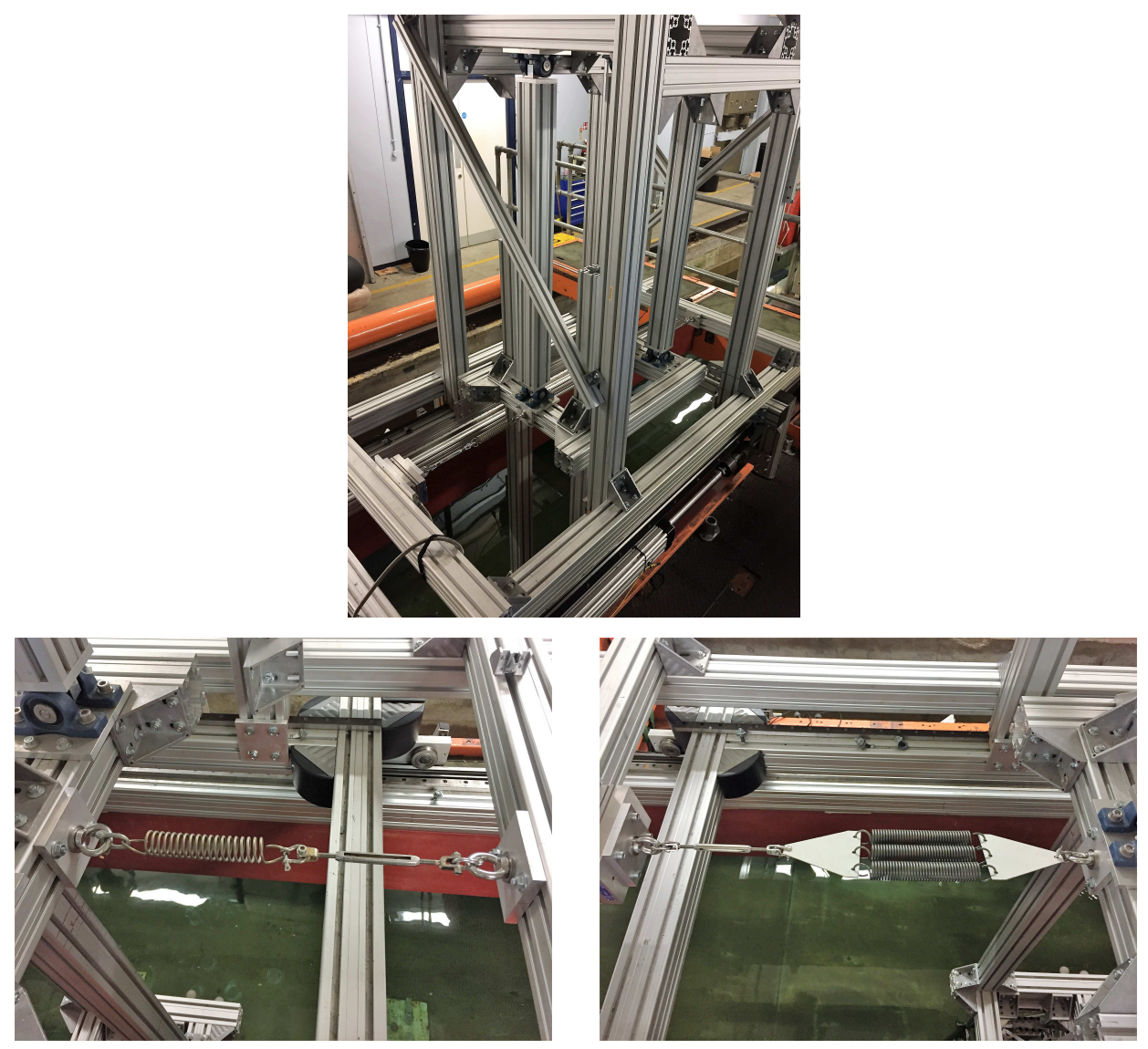

Figure 3: Top panel shows a photograph of the double pendulum setup on the carriage to support the jacket model. Also shown is the spring 1 arrangement being attached to the front and rear of the setup. Bottom panel shows photographs of the spring 1 arrangement at the rear of the setup (left), and the spring 2 at the front of the setup (right) which is then connected to a force transducer.

dominant wave frequency in order to represent both the second mode of compliant towers and the first mode of a jack-up leg. During the dynamic tests, the weaker springs result in relative jacket model displacement of $\sim \pm 1$ main leg diameter. Because of the parallel pendulum system, the jacket displacement is very close to uniform with water depth (horizontally) with negligible vertical excursion for small displacements of the order of 1 main leg diameter, as considered in this paper.

The same set of regular waves and wave groups as in Santo et al. (2018a) is used in these dynamic tests. To generate the localised wave groups, a set of 43 Fourier wave components was generated at the paddles following a JONSWAP-shaped amplitude spectrum truncated at $1 \mathrm{~Hz}$, 
with the frequency of the peak spectral energy at $0.52 \mathrm{~Hz}$ and a linear crest amplitude of $0.22 \mathrm{~m}$ at focus. The water depth is kept at $1.8 \mathrm{~m}$. Downstream along the tank, all the wave components are intended to come into focus which results in a perfectly focussed (defined as having a horizontal symmetry between the adjacent troughs either side of the largest crest in time). The same focussed wave group is also embedded into a set of regular wave backgrounds with wave heights of $0.1 \mathrm{~m}, 0.13$ $\mathrm{m}$ and $0.15 \mathrm{~m}$. It is worth noting, however, that in the tank the embedded focussed wave groups interacted with the background wave and the actual focus location was shifted downstream. This results in embedded wave groups being not perfectly focussed. This does not present significant difficulties for the comparison between the physical experimental forces and the CFD predictions, as iteration was used to ensure a good match between the measured and predicted incident waves at the model.

All the dynamic tests were done with one of three different towing speeds, representing uniform in-line (or following) current at the model: $0,0.14$ and $0.28 \mathrm{~m} / \mathrm{s}$, so the horizontal fluid velocity in the wave crests adds to the current. The same synchronisation system between the wave paddle and the carriage motion as previously used in the static tests as described by Santo et al. (2018a) is used throughout the dynamic tests. The synchronisation system is required to ensure that the jacket model towed under different speeds meets the same wave group at the right place and at the right time.

Apart from the dynamic tests, a repeat of the static tests was also conducted to provide reference data instead of re-using the previous results as described in Santo et al. (2018a). This is necessary because of a slight modification to the wave paddle system in the tank which causes difficulties to reproduce exactly the previous results of Santo et al. (2018a) (both in terms of surface elevation and force time histories).

\subsection{Data analysis}

In a situation where a structure is compliant and its displacement (which is uniform with depth and along the wave direction) is given by $x_{s}(t)$, the jacket motion can be assumed to satisfy the equation of motion for a single-degree-of-freedom (SDOF) mechanical oscillator, given by:

$$
m \ddot{x}_{s}(t)+c \dot{x}_{s}(t)+k x_{s}(t)=F(t)
$$

where $k$ is the spring stiffness, $c$ is the damping, $m$ is the mass of the system, and $F(t)$ is the external force acting on the jacket in time $t$. Here, we assume that the effective mechanical damping $c$ is 
linear, and that it can be estimated from a free vibration test in otherwise still water. Hence, this damping contains both fluid and structural components.

Assuming the Morison equation with the relative-velocity formulation is adequate to describe the hydrodynamic force, this can be expressed as (see e.g. Section 3.5 in Haritos (2007)):

$$
F(t)=\rho C_{m} V \dot{u}(t)-\rho C_{a} V \ddot{x}_{s}(t)+\frac{1}{2} \rho C_{d} A\left(u(t)-\dot{x}_{s}(t)\right)\left|u(t)-\dot{x}_{s}(t)\right|
$$

where $\rho$ is the water density, $V$ is the displaced volume of the structure, $u$ and $\dot{u}$ are the undisturbed flow velocity and acceleration, respectively, $A$ is the solid drag area, $C_{d}$ is the drag coefficient, $C_{m}$ is the inertia coefficient, and $C_{a}=C_{m}-1$ is the added mass coefficient.

Substituting Equation 2 into 3 and combining the $\ddot{x}_{s}(t)$ term, the following form can be obtained:

$$
\begin{gathered}
M \ddot{x}_{s}(t)+c \dot{x}_{s}(t)+k x_{s}(t)=F_{A}(t) \\
F_{A}(t)=F_{I}(t)+F_{D}(t) \\
F_{I}(t)=\rho C_{m} V \dot{u}(t) \\
F_{D}(t)=\frac{1}{2} \rho C_{d} A\left(u(t)-\dot{x}_{s}(t)\right)\left|u(t)-\dot{x}_{s}(t)\right|
\end{gathered}
$$

where $M=m+m^{\prime}$ is now the effective total mass of the system, $m^{\prime}=\rho C_{a} V$ is the added mass, $F_{A}(t)$ is the applied hydrodynamic force, which according to the Morison equation consists of a sum of the inertia force, $F_{I}(t)$ and the drag force, $F_{D}(t)$.

Equation 3 can be recast into the standard form:

$$
\ddot{x}_{s}(t)+2 \omega_{R} \zeta \dot{x}_{s}(t)+\omega_{R}^{2} x_{s}(t)=\frac{F_{A}(t)}{M}
$$

where $\zeta=c /\left(2 M \omega_{R}\right)$ is now the ratio of the actual to critical damping for the jacket in otherwise still water conditions, and $\omega_{R}=k / M$ is the natural (resonant) frequency of the whole system. This equation can be solved using an ordinary differential equation (ODE) solver with appropriate initial conditions.

In the physical tests, the measured force is obtained from a force transducer connected through a spring arrangement at the front face of the double pendulum setup. Thus, the measured force represents the horizontal reaction force to the ground $\left(F_{G}=k x_{s}\right)$ or equivalently the base shear. Since the actual model displacement $\left(x_{s}\right)$ is also measured using an optical tracking system, the same $F_{G}$ can also be derived from that. The actual hydrodynamic force $\left(F_{A}\right)$ applied onto the 
jacket model can be found by transforming Equation 3 into frequency domain as follows:

$$
\begin{aligned}
F_{A} & =k x_{s}+c \dot{x}_{s}+M \ddot{x}_{s} \\
\hat{f}_{A}(\omega) & =\left(k+i c \omega-M \omega^{2}\right) \hat{x}_{s}(\omega) \\
& =\left(1+i \frac{c}{k} \omega-\frac{M}{k} \omega^{2}\right) k \hat{x}_{s}(\omega) \\
\hat{f}_{A}(\omega) & =\left(1+i \frac{c}{k} \omega-\frac{M}{k} \omega^{2}\right) \hat{f}_{G}(\omega)
\end{aligned}
$$

Further substitution using $\zeta$ and $\omega_{R}$ into above equation yields:

$$
\hat{f}_{A}(\omega)=\left[1+i 2 \zeta\left(\frac{\omega}{\omega_{R}}\right)-\left(\frac{\omega}{\omega_{R}}\right)^{2}\right] \hat{f}_{G}(\omega)
$$

Equation 5 describes the transfer function (TF) between the measured force through the ground (or spring), $F_{G}$, and the applied force, $F_{A}$, in frequency domain. It is this inferred applied hydrodynamic force, $F_{A}$, that will be compared with numerical predictions, together with model displacement.

To estimate the natural frequency of the whole system due to different set of spring arrangement as well as damping rate important for dynamic analysis, a free vibration (push test) is conducted for each spring arrangement and the measured force is recorded. The natural frequencies due to spring 1 and 2 arrangement are found to be $0.988 \mathrm{~Hz}(6.209 \mathrm{rad} / \mathrm{s})$ and $1.272 \mathrm{~Hz}(7.989 \mathrm{rad} / \mathrm{s})$, respectively. Thus, for an incident wavegroup with peak frequency at $0.526 \mathrm{~Hz}$, the frequency ratio between the structural mode and the incoming wave is $\sim 1.9 \times$ and $\sim 2.4 \times$ for spring 1 and 2 arrangement, respectively.

There are two elements involved in the transfer function: the mass in the spring and the structural damping. The total mass can be measured/inferred, while the structural damping is fundamentally unknown but it will later be shown to be small compared to damping from (nonlinear) relative velocity contribution. What is left is the hydrodynamic damping, which consists of either linear (resulting from wave radiation and Stokes laminar boundary layers) or non-linear damping (vortex shedding, when the displacement is larger than the main cylinder diameter). Over the range of cases tested in the dynamic tests, the largest model displacement is of the order of $\sim \pm 1$ main leg diameter. Therefore, it is reasonable to assume a constant linear damping term as a model for the hydrodynamic damping, with the damping rate obtained from free oscillation tests (or push tests).

Figure 4 shows the envelopes of the recorded force time histories during free oscillations, which have been multiplied by an exponential term $\exp \left[0.0122 \omega_{R 1}(t-9)\right]$ and $\exp \left[0.0096 \omega_{R 2}(t-9)\right]$ for 


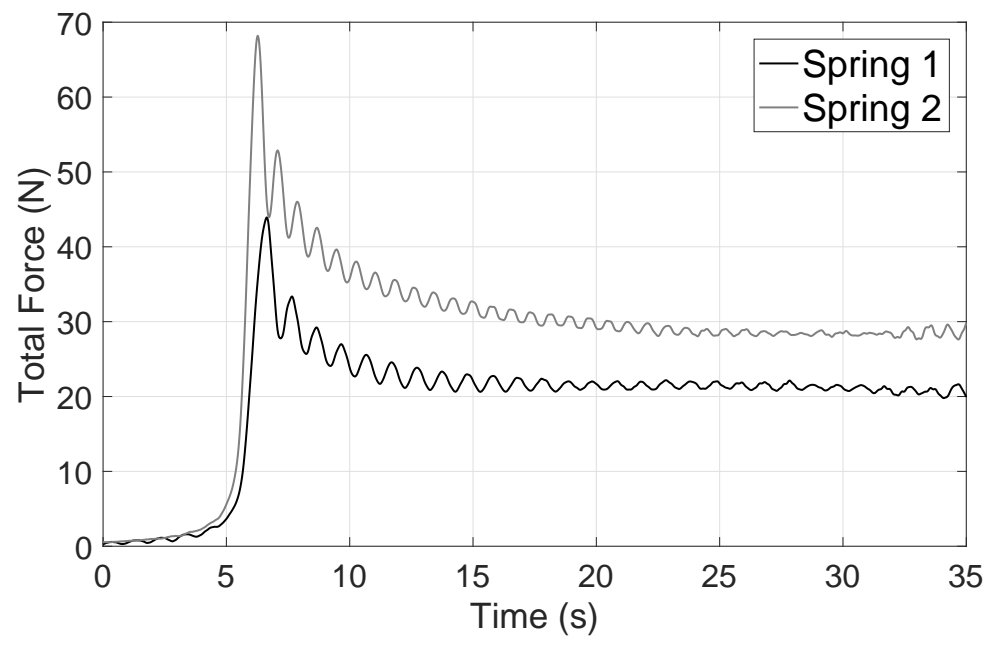

Figure 4: Envelopes of the recorded total force time histories measured through two different sets of springs during push test. These have been multiplied by $\exp \left[0.0122 \omega_{R 1}(t-9)\right]$ and $\exp \left[0.0096 \omega_{R 2}(t-9)\right]$ for spring 1 and spring 2 arrangement, respectively.

spring 1 and 2 arrangement, respectively. The oscillations at time $\sim 6$ sec are due to the starting transients and wrap-around effects of the signal processing in MATLAB ${ }^{\circledR}$. Most of the portions of the envelopes are close to horizontal, showing that the decay of the free oscillations of the jacket model in otherwise still water is close to linear with a non-dimensional damping coefficients of 0.0122 and 0.0096 for spring 1 and 2 arrangement, respectively. These structural damping rate values $\sim 1 \%$ will be used in the subsequent analysis, although they are lower than the API recommendation that the structural damping should be taken as $2-3 \%$ of critical for extreme wave analyses (API RP $2 \mathrm{~A}, 2000)$. Note that although the non-dimensional damping coefficients are different, the effective mechanical damping coefficient, $c=0.0122 \omega_{R 1}=0.0096 \omega_{R 2}$, is the same for both push tests, as one might expect if the damping occurs mostly outside the springs. As we shall see, much larger damping comes from the Morison relative-velocity term. Table 1 summarises the relevant parameters of the dynamic system.

\section{Numerical modelling and results}

\subsection{Numerical setup}

The numerical setup is similar to that reported by Santo et al. $(2015,2017)$ and recently by Santo et al. (2018a), using the same porous tower modelling approach with uniformly distributed 
Table 1: Summary of the dynamic system.

\begin{tabular}{lcc}
\hline Parameter & Spring 1 & Spring 2 \\
\hline$M$, Total mass (kg) & \multicolumn{2}{c}{152} \\
$k$, Stiffness $(\mathrm{N} / \mathrm{m})$ & 4600 & 8280 \\
$\zeta$, Damping relative to critical & 0.0122 & 0.0096 \\
$\omega_{R}$, Natural frequency $(\mathrm{Hz})$ & 6.209 & 7.989 \\
Frequency ratio & 1.9 & 2.4 \\
\hline
\end{tabular}

embedded Morison stresses. In essence, the stresses are distributed over the tower but expressed using the local (disturbed) flow kinematics, thus accounting for the global presence of the structure. The only difference is that in this paper the embedded Morison stresses are modified according to the Morison relative velocity formulation to account for the structural dynamic motion, see Equation 8.

The simulations are performed in the open source CFD code OpenFOAM ${ }^{\circledR}$ (http://www.openfoam.com) using the numerical wave tank 'waves2Foam' developed by Jacobsen et al. (2012). All the simulations are performed in two-phase flow (air and water) by solving the Reynolds-averaged NavierStokes equations coupled with the continuity equation for incompressible flows, and with an additional momentum sink term to account for the effect of the porous tower in the numerical simulation. The governing equations are written as:

$$
\nabla \cdot \mathbf{u}=0
$$

$$
\frac{\partial \rho \mathbf{u}}{\partial t}+\nabla \cdot\left[\rho \mathbf{u u}^{T}\right]=-\nabla p^{*}+\nabla \cdot[\mu \nabla \mathbf{u}+\rho \boldsymbol{\tau}]-\mathbf{S}+\left[-(\mathbf{g} \cdot \mathbf{x}) \nabla \rho+\sigma_{T} \kappa_{\gamma} \nabla \gamma\right]
$$

where $\rho$ is the fluid density, $\mathbf{g}$ is the acceleration due to gravity, $\mathbf{u}=(u, v, w)$ is the fluid velocity field in Cartesian coordinates, $p^{*}$ is the pressure in excess of hydrostatic pressure, defined as $p^{*}=$ $p-(\mathbf{g} \cdot \mathbf{x}) \rho, \mu$ is the dynamic viscosity, $\mathbf{x}=(x, y, z)$ is the local Cartesian coordinates, and $\boldsymbol{\tau}$ is the specific Reynolds stress tensor. The free surface (interface between air and water) is tracked using Volume-of-Fluid (VOF) method. The last two terms in Equation 7 in square brackets are for numerical convenience for the VOF method, and only active in the region where cell is partially filled with air, elsewhere these terms are zero. The $\sigma_{T} \kappa_{\gamma} \nabla \gamma$ term describes the surface tension 
effect using the CSF (Continuum Surface Force) model of Brackbill et al. (1992), where $\sigma_{T}$ is the surface tension coefficient, and $\kappa_{\gamma}$ is the surface curvature. $\gamma$ is a scalar field used to represent the fraction of a cell volume filled with water (interface), with $0 \leq \gamma \leq 1$; 0 for air, and 1 for water. In the numerical simulation, the interface value of 0.5 and greater is treated as the water phase. For more details of the interface treatment, see Berberović et al. (2009).

A sink term is used to account for momentum lost from the flow, which in the case of a simple homogeneous porous tower modified according to the relative velocity version of the Morison equation (see e.g. Section 3.5 in Haritos (2007)) is written as:

$$
\mathbf{S}=\frac{1}{2} \rho F\left(\mathbf{u}-\mathbf{u}_{\mathbf{s}}\right)\left|\mathbf{u}-\mathbf{u}_{\mathbf{s}}\right|+C_{m}^{\prime} \frac{\partial \rho \mathbf{u}}{\partial t}
$$

where $\mathbf{u}_{\mathbf{s}}$ is the structural velocity (note that this is equal to $\dot{x}_{s}(t)$ in the structural dynamics formulation, and is constant in space over the entire block), $F$ is the Forchheimer resistance parameter, and $C_{m}^{\prime}$ is the equivalent of the local Morison inertia coefficient, $C_{m}$, but here defined in the porous tower context.

Although the jacket model is made to undergo free vibration in the actual dynamic tests, there is no moving (or dynamic) mesh involved in the numerical modelling. Instead a time-varying stress is implemented according to Equation 8 and the governing equation is solved with a static computational mesh domain.

Three methods are available to solve for the modified flow field accounting for the structural velocity (or motion), outlined as follows:

- Method 1. To couple an ODE solver (with initial conditions for displacement and velocity) with CFD to solve internally (with a feedback loop) for the predicted total force and model displacement (and velocity). The predicted force and displacement will be directly compared with measurements. This is the most complete and integrated Method that solves the coupled system with the least number of assumptions. However, we note that for a real structure, Method 1 would require dynamic coupling between two complex computational codes, for instance OpenFOAM and a structural dynamics code such as USFOS ${ }^{\circledR}$ (www.usfos.no), such that the information is continuously passed to and fro.

- Method 2. To use an approximate expanded form of the Morison relative-velocity following Haritos (2007) and Merz et al. (2009) to solve for the model displacement (and velocity) 
and subsequently the predicted total force. This will become our recommended Method for practical industry applications. No dynamic coupling is required. The output of fluid loading calculation from CFD can be stored and then read in as required by the structural code.

- Method 3. To read in $\mathbf{u}_{\mathbf{s}}$ as an external input from the measurement (structural displacement converted to velocity) into CFD to solve for the predicted total force, to be compared with measured total force. The predicted force is then convoluted with the same transfer function (TF) in the frequency domain to yield predicted model displacement, to be compared with measured displacement. This Method serves as an internal check for consistency on Method 1.

The detailed approach of Method 2 is presented as follows. Referring back to Equation 3, in the case when $\dot{x}_{s}(t)$ is small compared to $u(t)$ which is true for all the practical cases, an approximation for the Morison relative-velocity term in the drag force is possible as shown by Haritos (2007) and Merz et al. (2009). This has the form:

$$
\begin{aligned}
F_{D}(t) & =\frac{1}{2} \rho C_{d} A\left(u(t)-\dot{x}_{s}(t)\right)\left|u(t)-\dot{x}_{s}(t)\right| \\
& \approx \frac{1}{2} \rho C_{d} A\left(u(t)|u(t)|-2|u(t)| \dot{x}_{s}(t)\right)
\end{aligned}
$$

Figure 13 in Appendix illustrates the very small difference between the above two expressions for realistic flow and structural velocities. The approximate form allows the structural velocity term to be de-coupled from the hydrodynamic calculation. Under these circumstances, Equation 3 can be simplified as shown by Haritos (2007) as:

$$
\ddot{x}_{s}(t)+2 \omega_{R}\left(\zeta+\zeta_{H}(t)\right) \dot{x}_{s}(t)+\omega_{R}^{2} x_{s}(t)=\frac{F_{I}(t)+F_{D}^{\prime}(t)}{M}
$$

where

$$
F_{D}^{\prime}(t)=\frac{1}{2} \rho C_{d} A u(t)|u(t)|
$$

which is simply the static drag force (in the case of rigid support conditions), and

$$
\zeta_{H}(t)=\frac{\frac{1}{2} \rho C_{d} A|u(t)|}{M \omega_{R}}
$$

which is the contribution to damping from the hydrodynamic drag interaction term, or the Morison wave-current blockage term. Therefore, Method 2 involves using just the information (disturbed 
kinematics accounting for wave-current blockage) from the static structure simulations to solve (as post-processing) for the model displacement (and velocity) via a time-domain ODE solver, and subsequently the actual applied dynamic force, all accounting for wave-current-structure blockage. Therefore, the need to run the CFD code simultaneously with dynamic transfer of information in both directions with a sophisticated structural dynamics code such as USFOS can be avoided, which from the practical application renders Method 2 rather attractive.

Results from the three methods will be presented in the next subsection. For comparison with static tests, the same governing equation are solved but without the $\mathbf{u}_{\mathbf{s}}$ contribution.

A porous tower having the same physical dimensions, the amount of resistance and the added mass of the actual jacket is modelled. Following Santo et al. (2014a, 2015), the following relationship holds for the calibration of $F$ and $C_{m}^{\prime}: C_{d} A / A_{f}=F L, C_{m}^{\prime}=C_{m} V / V_{P}$ and likewise for $C_{a}^{\prime}$, where $A$ and $A_{f}$ are the solid drag area and the frontal area of the actual jacket model, respectively, $L$ is the downstream length of the jacket model as well as the porous block, $V$ is the displaced volume of the elements in the jacket model, and $V_{P}=A_{f} \times L$ is the enclosed volume of the porous tower. $C_{m}$ is the Morison inertia coefficient, and $C_{d}$ is the drag coefficient. For our jacket model shown in Figure 2, the actual total values of $A, A_{f}$ and $V$ compatible for use in the standard Morison formulation are $1.17 \mathrm{~m}^{2}, 0.57 \mathrm{~m}^{2}$, and $0.024 \mathrm{~m}^{3}$, respectively, all measured from the bottom of the model up to $0.25 \mathrm{~m}$ above still water level. Hence, both $L$ and $V_{p}$ of the porous tower are based on the actual geometry of the jacket model. The sensitivity of the results to the precise geometric arrangement of the tower has been assessed previously in Santo et al. (2018a). The same paper also discusses the key similarities and differences between the physical experiments and the numerical simulations.

The same numerical domain taking the advantage of one-way information transfer as described previously in Santo et al. (2018a) is used throughout the analysis. On average, each 3D simulation with 5.2 million cells took $\sim 7$ days for a 15 wave period run on 24 processors. All simulations were run on the High Performance Computing (HPC) facilities of the National University of Singapore.

\subsection{Results}

Here we present selected results in terms of composite plots of surface elevation (measured), hydrodynamic force from static test (measured), model displacement (measured), base shear (measured), to hydrodynamic force from dynamic test (inferred using the Transfer Function in Equa- 
tion 5). We also present comparisons between measurements and numerical predictions for each of the selected cases. The aim is to demonstrate that same approach works well for the different cases, which justifies the robustness of our experimental and numerical modelling approach. We emphasise that all the numerical results are obtained using $C_{d}=1.3$ and $C_{m}=2.0$, consistent with the previous results of Santo et al. (2018a). Unless otherwise stated, the numerical results are presented using Method 1 throughout the rest of the paper.

Figure 5 presents such comparisons with the spring 1 arrangement, and Figure 6 is for spring 2 , for a range of cases from an isolated focussed wave group without current to an embedded wave group in a regular wave background with a significant current. In general, good agrement in terms of the incident surface elevations is obtained, showing that we have been able to reproduce numerically the incident undisturbed wavefield from the experiments. Going from the kinematics to the applied hydrodynamic force in the static tests, relatively good agreement is observed for the complete force time history, consistent with the previous results of Santo et al. (2018a). Including now the effect of structural motions, the comparison is such that the hydrodynamic forces from dynamic tests are generally slightly over-predicted, which then result in a slight under-estimation of the model displacements. In general, the peak-to-peak response (both in terms of model displacement and applied force) is reasonably well captured numerically for each of the two different frequency ratios. This demonstrates the validity of the Morison relative-velocity formulation, cast in terms of global wave-current-structure blockage for dynamically-responding structures in a high frequency mode of vibration. Given the slight conservatism of the numerical results, we view this level of agreement as satisfactory.

The numerical modelling presented so far can adequately capture the complete hydrodynamic force and model displacement time histories, although the model displacement is uniform with water depth here for simplicity. For a practical application with a realistic space-frame structure having a model displacement field which is varying with water depth, simultaneous modal decomposition needs to be included in the time-domain ODE solver to allow for multiple modes of tower vibration. Although this is perfectly possible, the detailed implementation could be slightly more complicated. Also, to couple a CFD solver, such as OpenFOAM, with a full structural dynamics code, such as USFOS, is definitely non-trivial. Hence, in terms of practical industry use, the application of Method 1 is perhaps rather limited. Therefore, it is of interest to investigate whether an approximation to the Morison relative-velocity term is valid, which is the Method 2. 

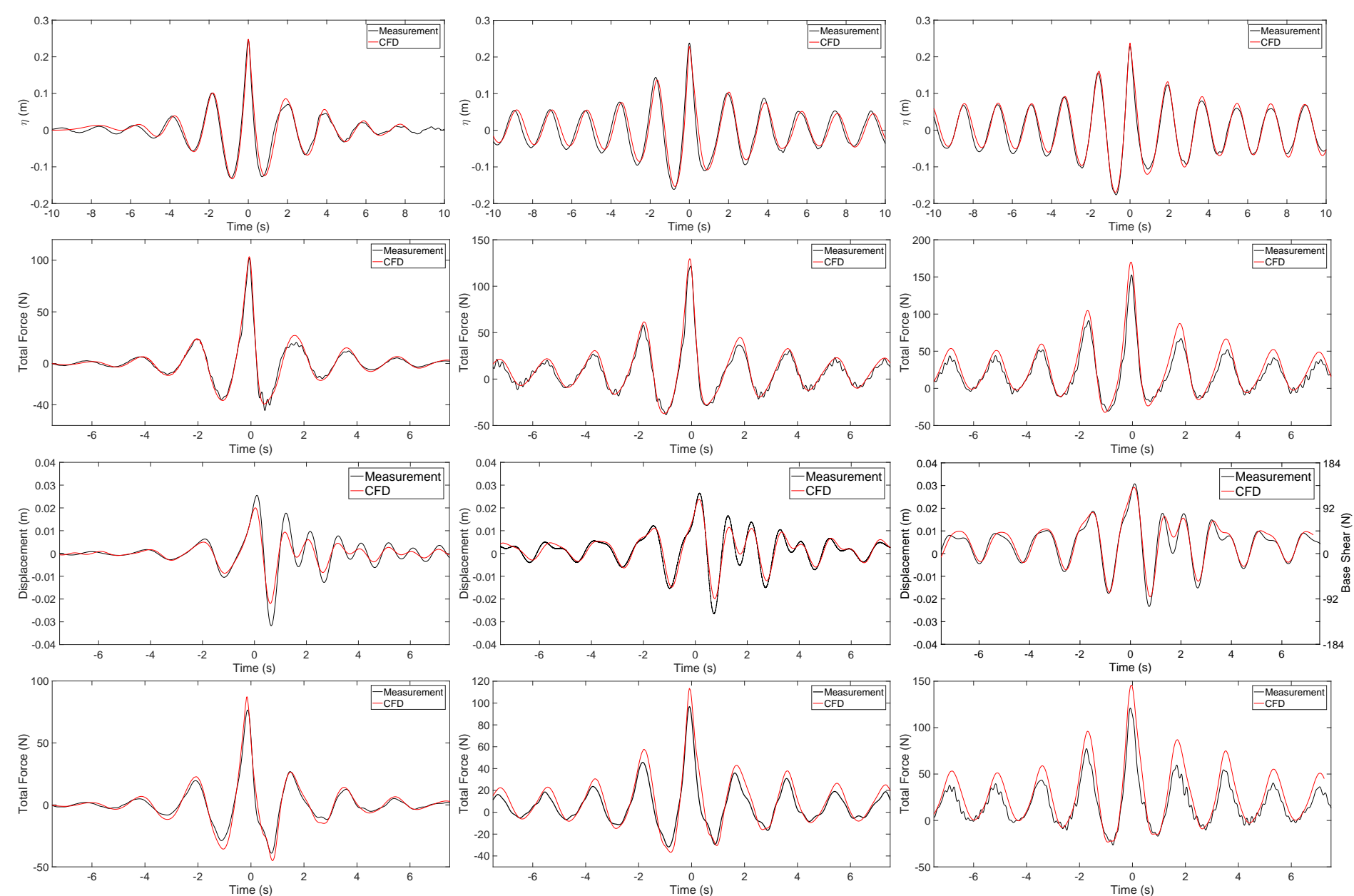

Figure 5: Comparison between numerical predictions and measurements in terms of: surface elevation (top row), total hydrodynamic force from static tests (second row), model displacement (third row), and total hydrodynamic force from dynamic tests with spring 1 arrangement (bottom row). The base shear at the right axis of model displacement is the reaction force to ground or spring force. The numerical results for the dynamic tests are obtained using Method 1 as described in the main text. Three cases are presented: a focussed wave group without current (left panels), an embedded focussed wave group in $0.1 \mathrm{~m}$ regular wave background with $0.14 \mathrm{~m} / \mathrm{s}$ current (middle panels), and an embedded focussed wave group in $0.13 \mathrm{~m}$ regular wave background with $0.28 \mathrm{~m} / \mathrm{s}$ current (right panels). 

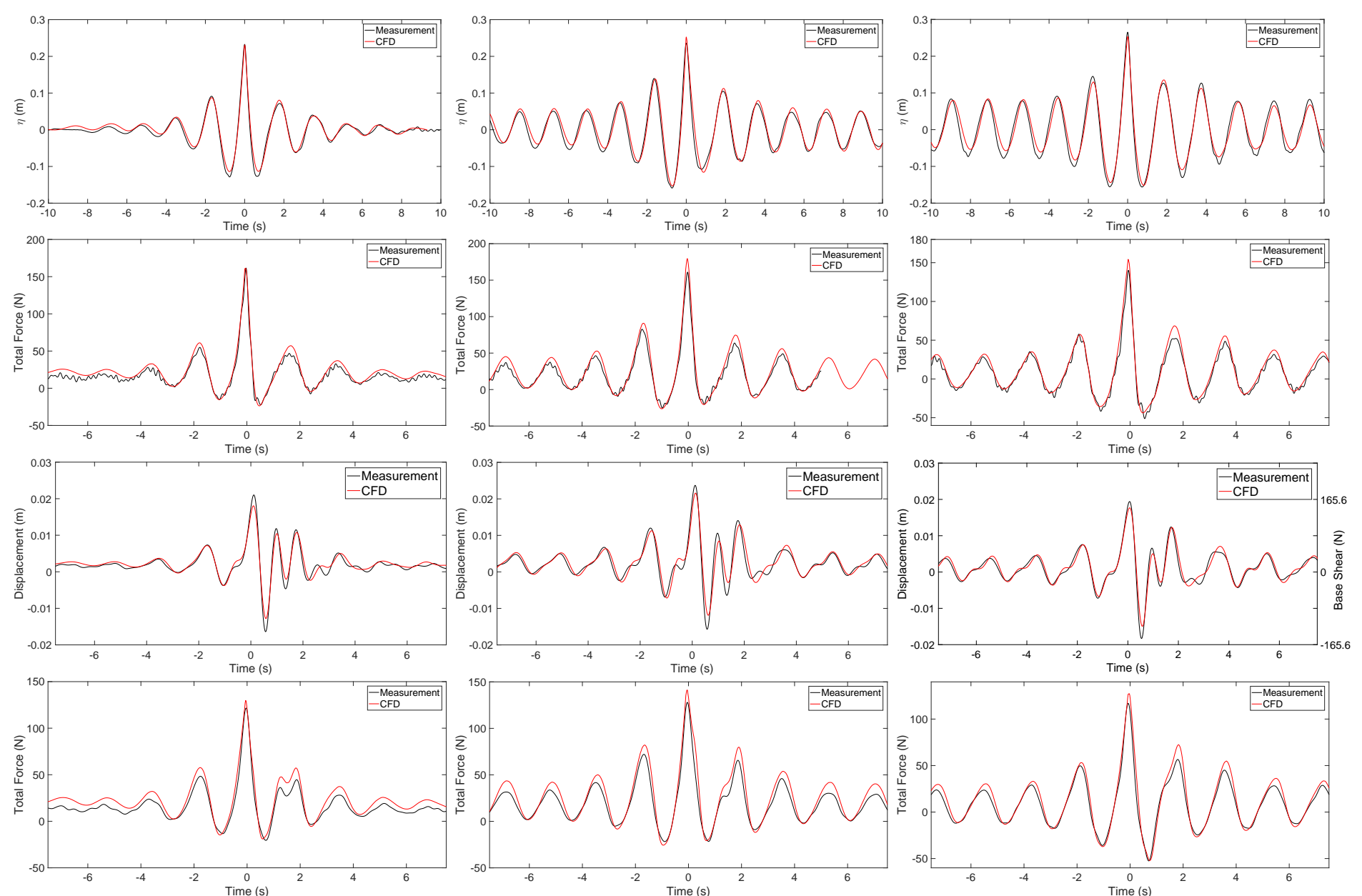

Figure 6: Comparison between numerical predictions and measurements in terms of: surface elevation (top row), total hydrodynamic force from static tests (second row), model displacement (third row), and total hydrodynamic force from dynamic tests with spring 2 arrangement (bottom row). The base shear at the right axis of model displacement is the reaction force to ground or spring force. The numerical results for the dynamic tests are obtained using Method 1 as described in the main text. Three cases are presented: a focussed wave group with $0.28 \mathrm{~m} / \mathrm{s}$ current (left panels), an embedded focussed wave group in $0.1 \mathrm{~m}$ regular wave background with $0.28 \mathrm{~m} / \mathrm{s}$ current (middle panels), and a $180^{\circ}$ phase shift to embedded wave group in $0.15 \mathrm{~m}$ regular wave with $0.14 \mathrm{~m} / \mathrm{s}$ current (right panels). 
We examine the adequacy of the form by simply using the modified (or disturbed) kinematics from numerical static structure runs to post-process the applied static force and the additional damping from Morison relative-velocity following Equation 10 and 11. We then solve for the model displacement (and velocity) by time-marching Equation 10 using a time-domain ODE solver (using a ode45 solver in MATLAB). The applied dynamic force can then be predicted using Equation 9 once the model velocity is obtained. We perform this for a range of cases as shown in Figure 7 together with the numerical predictions obtained from Method 1. It is worth stressing that the approximate form is valid so long as $\dot{x}_{s}(t)$ is small compared to $u(t)$, however since $u(t)$ crosses through zero over time, there are parts of the load cycle where the condition is not strictly valid. Nevertheless, those parts of the load cycle are dominated by inertia force instead, therefore overall the approximate form works well. Good agreement between the two Methods can be seen in general in terms of both the model displacement and the applied dynamic force time histories, which demonstrates that the approximate form is valid for the practical range of high frequency mode of vibrations considered in this paper. Although low frequency mode of vibration was not considered in this paper, the structural velocity is also always smaller than the disturbed flow velocity. This generalises Method 2 to cover across both high and low frequency modes of compliant towers. From the practical industry application, Method 2 is very useful since the need to dynamically couple the CFD code with a structural dynamics model can be avoided.

We now compare the numerical predictions obtained using Method 1 and 3 specifically in terms of hydrodynamic forces, all relative to measurement, for a range of cases as shown in Figure 8. Good agreement between numerical predictions and measurements in terms of surface elevations is obtained. It is worth noting that Method 3 uses the measured structural velocity to solve for the modified flow field accounting for blockage effect. Therefore, the underlying assumption is that the applied hydrodynamic force on the jacket model can be reasonably well reproduced numerically, which has been demonstrated from the static force comparison in the previous figures. The predicted model displacement from Method 3 can be solved by combining the predicted forces with the same Transfer Function (Equation 5), hence requiring a two-step approach. The comparison in terms of model displacement is reasonable, given that in general the displacement is slightly over-estimated. The slight over-estimation and the high frequency oscillation away from the main response is attributed to the finite length effect of the numerical results, which are in general of shorter timescale than the measurements due to the use of limited simulation times and truncated 

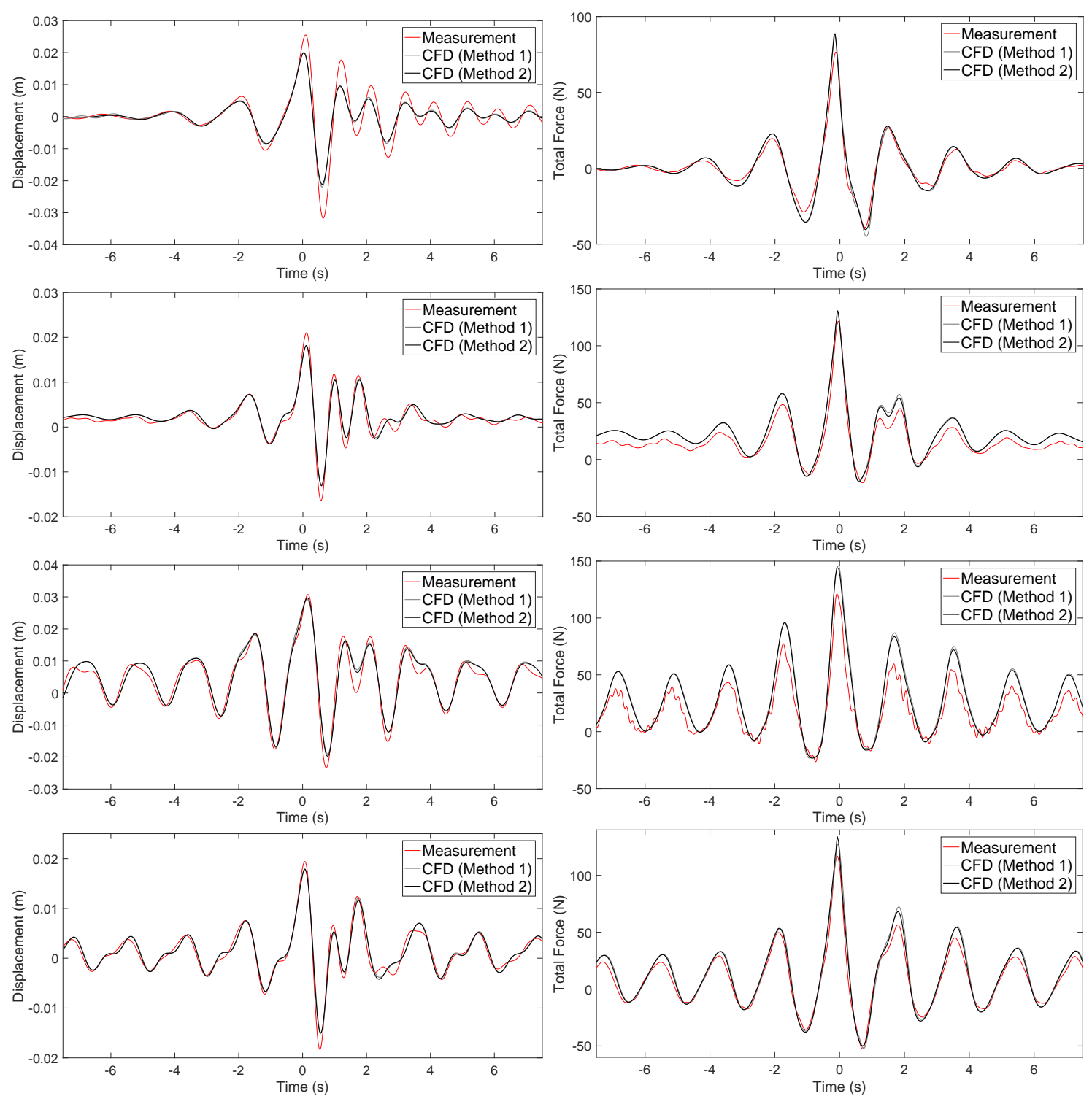

Figure 7: Comparison of model displacements (left) and applied hydrodynamic force time histories (right) between numerical predictions obtained from Method 1 and 2, all relative to measurements. Four cases are presented: a focussed wave group without current with spring 1 arrangement (top row), the same wave group with $0.28 \mathrm{~m} / \mathrm{s}$ current with spring 2 arrangement (second row), an embedded wave group in $0.13 \mathrm{~m}$ regular wave with $0.28 \mathrm{~m} / \mathrm{s}$ current with spring 1 arrangement (third row), and a $180^{\circ}$ phase shift to embedded wave group in $0.15 \mathrm{~m}$ regular wave with $0.14 \mathrm{~m} / \mathrm{s}$ current with spring 2 arrangement (bottom row). 

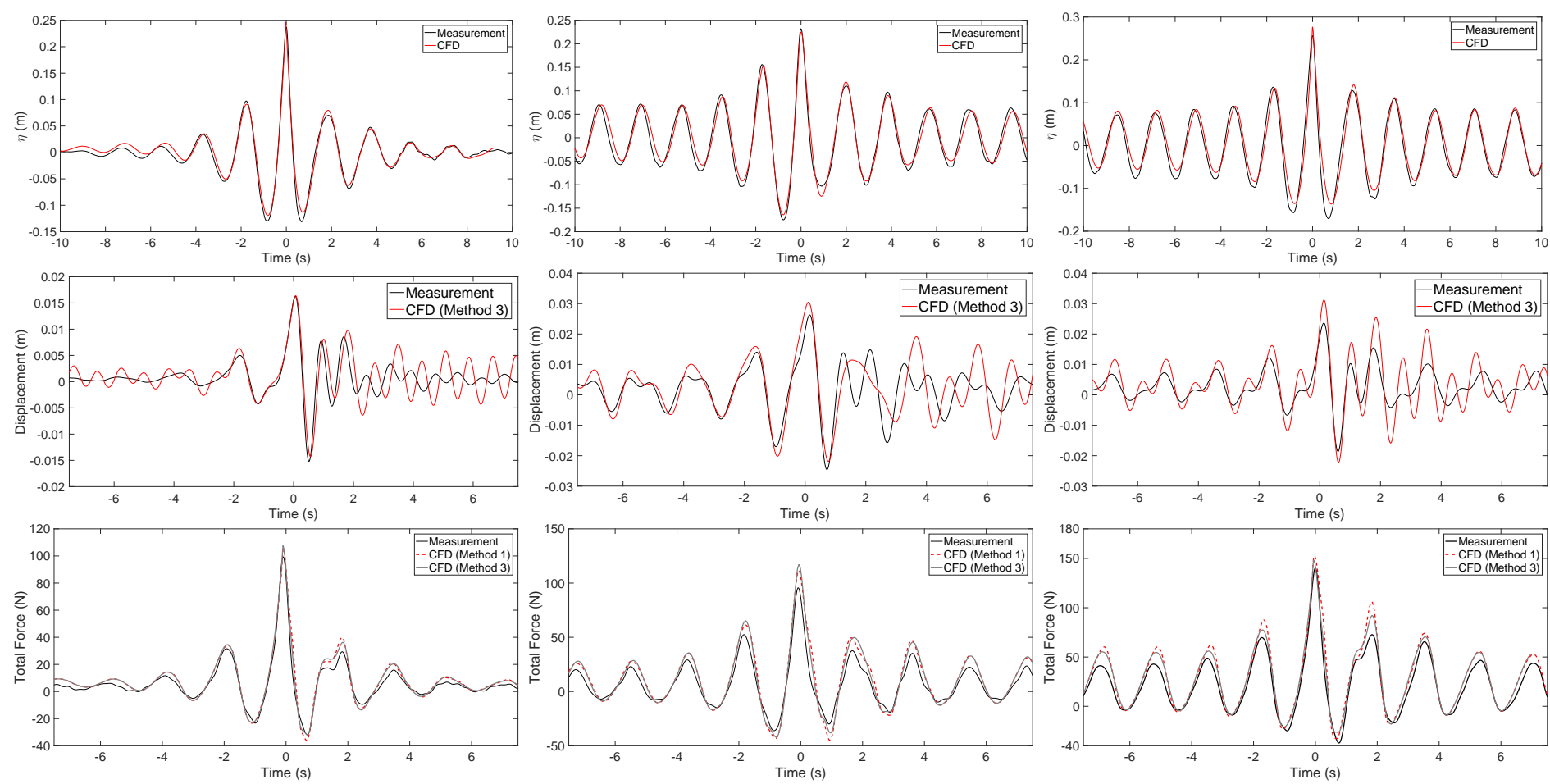

Figure 8: Comparison between numerical predictions obtained from Method 3 and 1 and measurements in terms of: surface elevation (top row), model displacement (middle row), and total force from dynamic tests with spring 1 or 2 arrangement (bottom row). Three cases are presented: a focussed wave group with $0.14 \mathrm{~m} / \mathrm{s}$ current with spring 2 arrangement (left panel), an embedded focussed wave group in $0.13 \mathrm{~m}$ regular wave background with $0.14 \mathrm{~m} / \mathrm{s}$ current with spring 1 arrangement (middle panel), and a $180^{\circ}$ phase shift to embedded wave group in $0.15 \mathrm{~m}$ regular wave with $0.28 \mathrm{~m} / \mathrm{s}$ current with spring 2 arrangement (right panel). 
domains to reduce computational effort. In terms of hydrodynamic forces, relatively good agreement between the two numerical methods demonstrate the robustness of the proposed fluid loading recipe using CFD with porous tower modelling approach.

Overall, reasonably good agreement is obtained between numerical predictions and measurement for a range of cases tested in the dynamic tests all using a single set of Morison coefficients $C_{d}=1.3$ and $C_{m}=2.0$, with the numerical predictions resulting in slight over-estimation in the predicted applied hydrodynamic forces. This is considered satisfactory as there is a degree of conservatism built into the numerical modelling using the Morison with relative velocity formulation accounting for wave-current-structure blockage effects.

\section{Discussions}

\subsection{On the summary of the comparison}

Figure 9 presents a composite plot summarising the comparison between measurements (left column) and numerical predictions (right column) for the case of a focussed wave group with crest height of $0.25 \mathrm{~m}$ and $0.28 \mathrm{~m} / \mathrm{s}$ current with static, spring 1 and 2 arrangements. The top row shows the comparison in terms of total applied hydrodynamic force. Going from statically-responding to dynamically-responding cases, one can see the reduction in the peak hydrodynamic force as the dynamic system experiences reduced drag due to the structure motion. The force difference relative to the static case is the contribution from the relative velocity which can be viewed as an extra damping term (see Equation 11). The bottom row shows the comparison in terms of force to the ground, or base shear. The base shear of the dynamically-responding cases is observed to be larger than that of the statically-responding case, a manifestation of a dynamic amplification factor as a result of the structural dynamics. For all cases, the numerical predictions match reasonably well with the measurements, demonstrating the adequacy and robustness of the proposed approach in capturing the global hydrodynamic loading on both statically- and dynamically-responding spaceframe structures.

\subsection{On the importance of additional damping from the relative velocity}

An important question remains as to how much additional damping comes from the Morison relative-velocity term relative to the existing structural and assumed linear hydrodynamic damping estimated from the push tests. This can be assessed by using the hydrodynamic force from the 

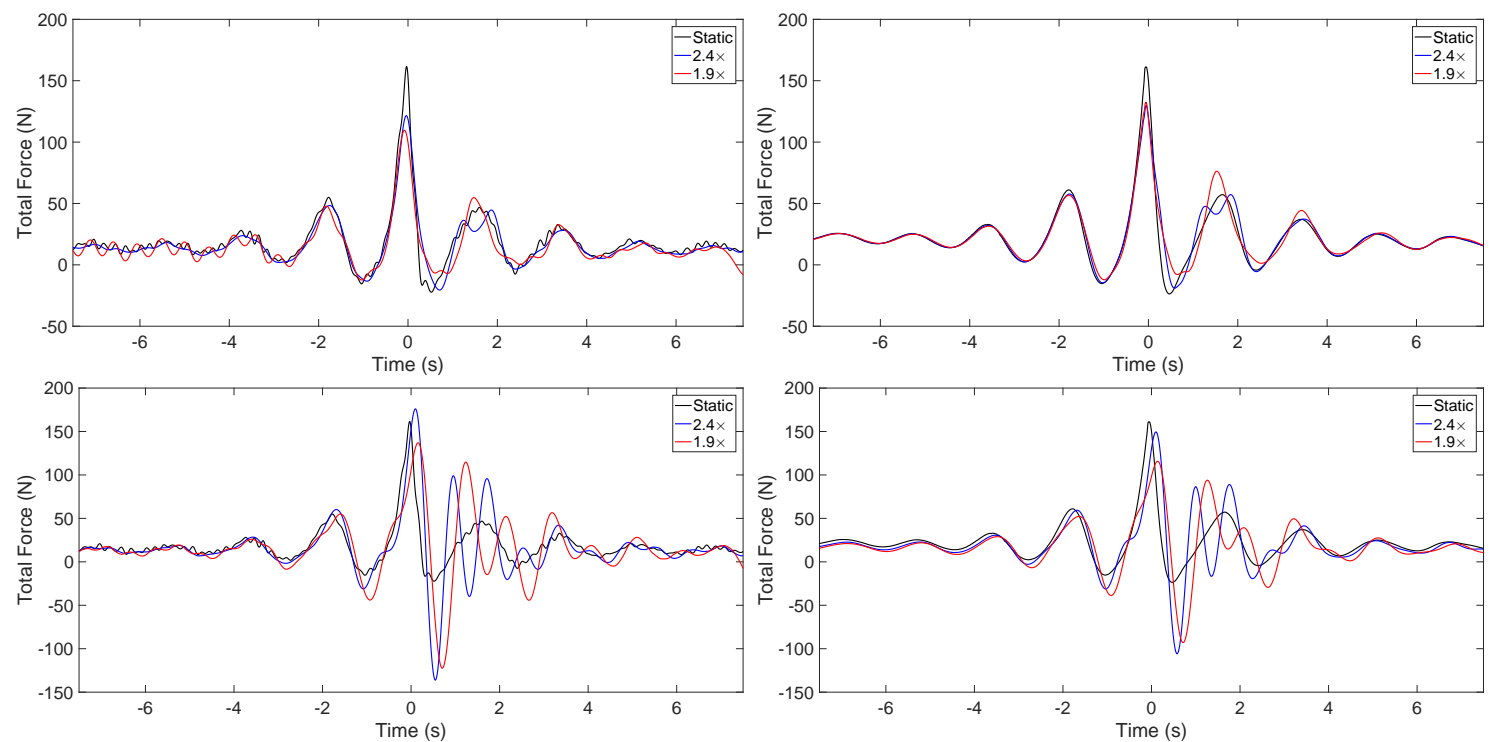

Figure 9: Comparison between measurements (left column) and numerical predictions (right column) in terms of: total applied hydrodynamic force (top row), and base shear or force to ground (bottom row) for the case of a focussed wave group with $0.28 \mathrm{~m} / \mathrm{s}$ current with static, spring 1 and 2 arrangement.

static tests to drive a time domain model using an ODE solver to establish the amount of damping rate needed in order for the predicted displacement to reasonably fit well the entire measured displacement from the dynamic tests. Figure 10 demonstrates such analysis using the predicted (static) forces from CFD and an artificial damping rate of $7 \times \zeta$, which is equivalent to structural damping of $8 \%$ of critical.

In general, good agreement between the measurements and the numerical predictions can be obtained for a range of cases without and with different currents and regular wave backgrounds. For an isolated wave group with no wave background and no current, eventually as the wave stops the damping must look like the push-test behaviour, hence the slower decay of the actual measured displacement relative to the predicted one as obvious from Figure 10 (top panel). With the background waves and/or a mean current present, the damping will remain much higher, demonstrated by the good agreement even after the main wave group has passed by as shown in Figure 10 (middle and bottom panel). The universality of the results, regardless of current and wave condition, demonstrates that the Morison with relative velocity term, coupled with blockage effects to some extent, contributes to significant additional damping into the dynamic system. 

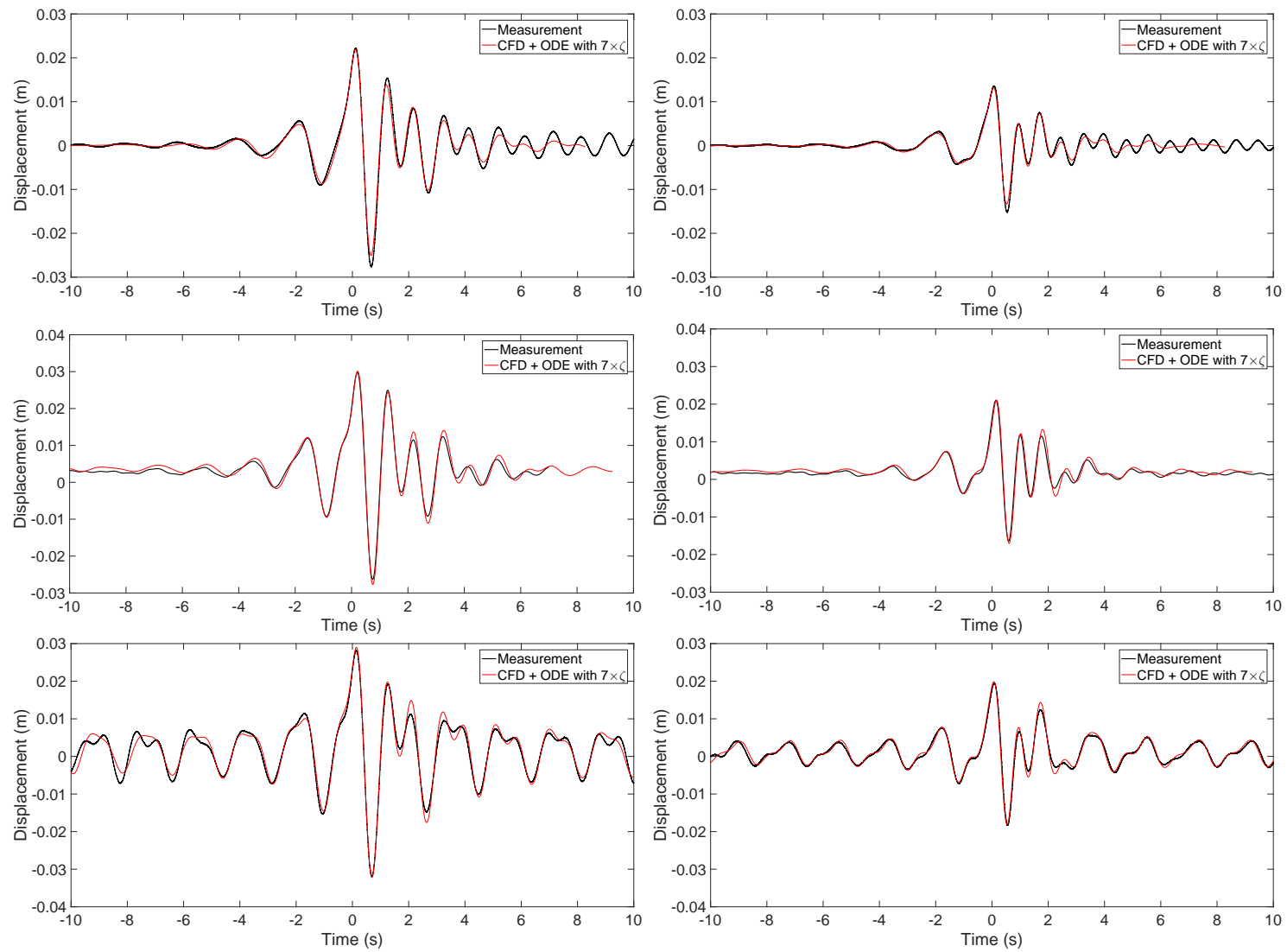

Figure 10: Comparison of model displacement time histories between numerical predictions (red) and measurements (black) from dynamic tests from dynamic tests with spring 1 (left) and spring 2 (right) arrangement for three cases. The numerical results are obtained from applying the predicted static force from CFD into an external ODE model (in MATLAB) with a damping rate of $7 \times \zeta$. Top row is for a focussed wave group without current. Middle row is for the same focussed wave group with $0.28 \mathrm{~m} / \mathrm{s}$ current. Bottom row is for a $180^{\circ}$ phase shift to embedded wave group in $0.15 \mathrm{~m}$ regular wave with $0.14 \mathrm{~m} / \mathrm{s}$ current.

It is thus of interest to perform CFD simulations with the actual structural damping rate set to zero $(\zeta=0)$. Figure 11 presents the results obtained from either Method 1 or 2 in terms of model displacement and hydrodynamic force time histories between $\zeta \neq 0$ and $\zeta=0$, all relative to the measurements. It is obvious from the figure that the numerical results using the two different $\zeta$ values for the structural damping are virtually indistinguishable, which supports the observation that the additional damping from the relative velocity dominates completely the existing structural and linear hydrodynamic damping estimated from the push tests. From the practical point of view, 

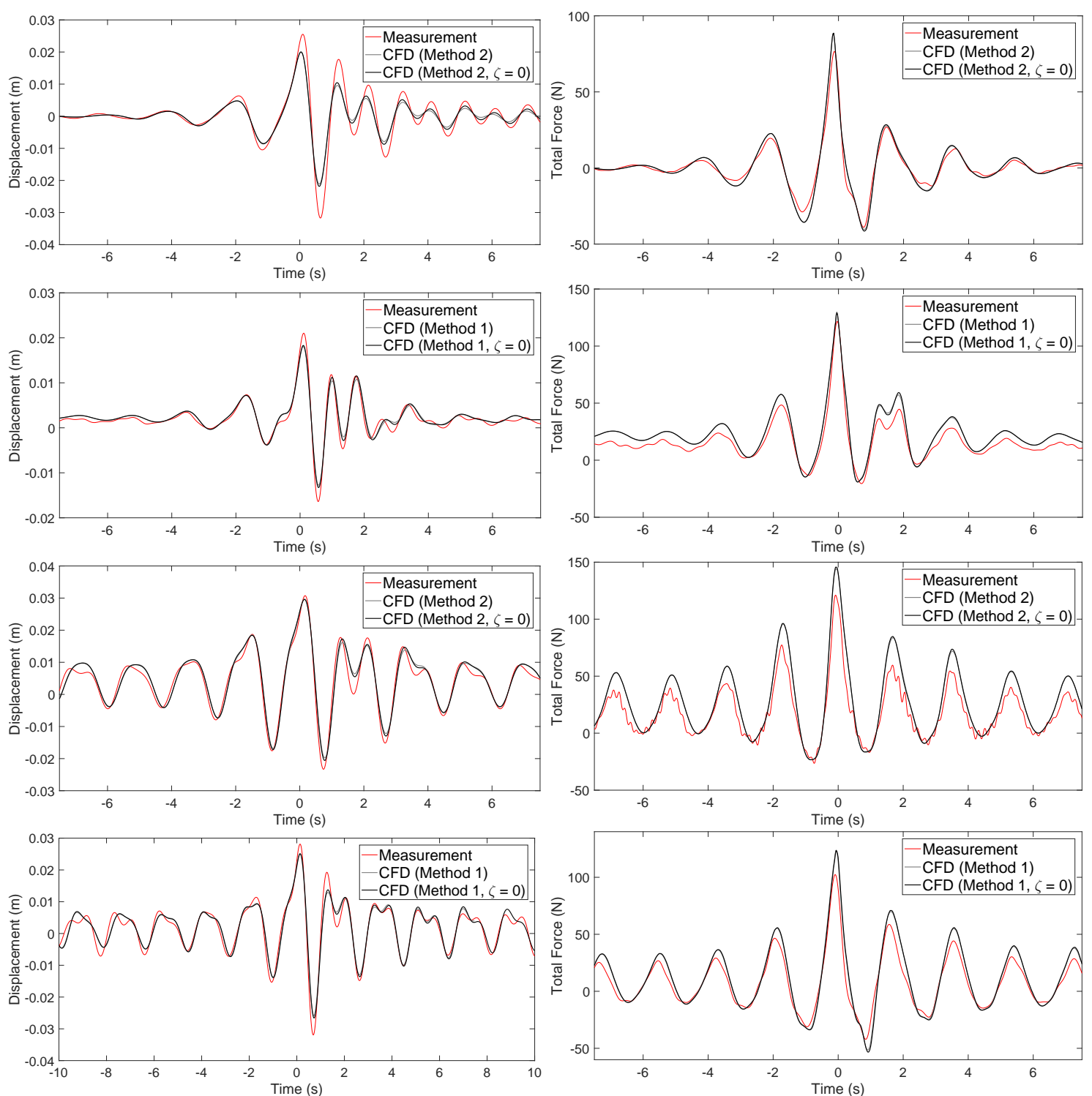

Figure 11: Comparison between numerical predictions (obtained from either Method 1 or 2) using the actual measured $\zeta$ and $\zeta=0$ relative to measurements in terms of: model displacement (left panel), and total force from dynamic tests with spring 1 or 2 arrangement (right panel). Three cases are presented: a focussed wave group without current with spring 1 arrangement (top row), a focussed wave group with $0.28 \mathrm{~m} / \mathrm{s}$ current with spring 2 arrangement (middle row), and a $180^{\circ}$ phase shift to embedded wave group in $0.15 \mathrm{~m}$ regular wave with $0.14 \mathrm{~m} / \mathrm{s}$ current with spring 1 arrangement (bottom row). 

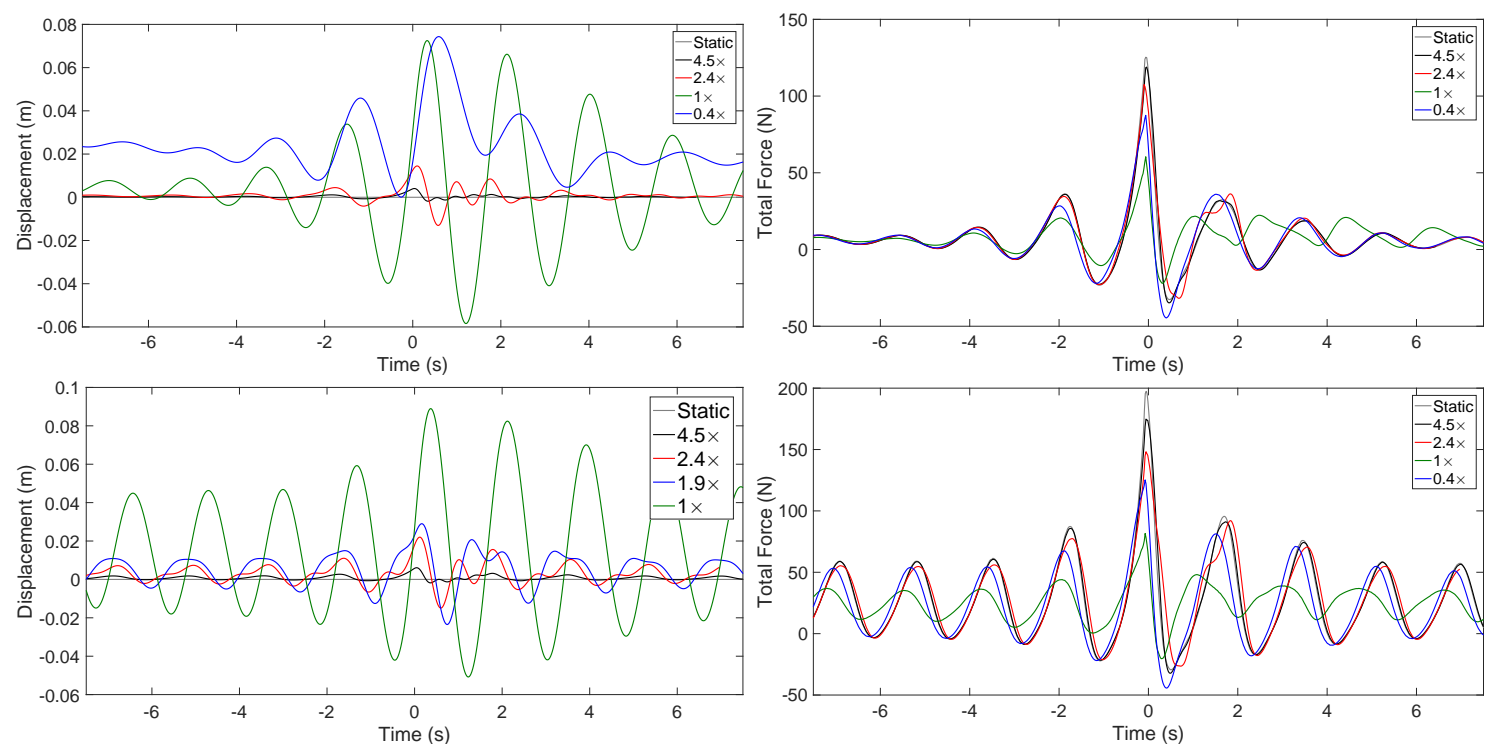

Figure 12: Comparison among numerical predictions with different spring arrangements producing high frequency ratios approaching static (rigid) case in terms of model displacement (left panel), and total hydrodynamic force (right panel). Two cases are presented: a focussed wave group with $0.14 \mathrm{~m} / \mathrm{s}$ current (top row), and a $180^{\circ}$ phase shift to embedded wave group in $0.15 \mathrm{~m}$ regular wave with $0.28 \mathrm{~m} / \mathrm{s}$ current (bottom row).

this result is convenient because the uncertainty in the assessment of the structural damping term can now be ignored.

\subsection{On other frequency ratio cases, including a statically-responding (stiff) structure}

Given the validity of the numerical modelling, it is also of interest to numerically investigate flow regimes with frequency ratios different to those tested in the physical experiments, since in the higher frequency ratio limit the structural response and applied force should approach those of a statically-responding (rigidly supported) structure. This is examined for two cases as shown in Figure 12. Apart from the two frequency ratio cases of $1.9 \times$ and $2.4 \times$ actually tested in the towing tank, additional frequency ratios of $0.4 \times, 1 \times$, and $4.5 \times$ are included together with the static case (where the frequency ratio $\rightarrow \infty$ in principle).

It can be seen that as the frequency ratio increases approaching the static case limit, the model response in terms of model displacement and applied force time histories also approach the response of the static case. This demonstrates the consistency in our numerical modelling approach. 


\subsection{On the scaling effects}

For a realistic jacket model geometry with a scale of 1:80 as considered in this paper, at field scale the peak crest of a large wave becomes $\sim 20 \mathrm{~m}$ with an in-line current of $2.5 \mathrm{~m} / \mathrm{s}$ using Froude scaling. Also, the resultant total hydrodynamic force becomes $\sim 50-100 \mathrm{MN}$, and the peak structural displacement is $\sim 3 \mathrm{~m}$. The values of the peak displacement are plausible and comparable to the second mode of an actual compliant tower, but it is larger than a typical first mode of an actual jack-up legs $(\sim 0.5-1 \mathrm{~m})$. Even for a frequency ratio of $1 \times$, the peak displacement is $\sim 7 \mathrm{~m}$, which might not be catastrophic. The main question remains as to whether this increased damping due to relative-velocity will be present in reality for the full-scale flow regime, and if so whether it will scale appropriately. We believe the only change from lab to field scale would be on the selection of suitable Morison coefficients, where guidance from API RP 2A (2000) is useful, and the necessary inclusion of a full structural dynamics representation of the structure with a code such as USFOS.

It is worth noting that the optimum $C_{d}$ which gives the best fit to the measured drag is found to be $\sim 1.3$; high but reasonable since we do not account for local velocity amplification due to the presence of other members, in particular due to the closely-spaced conductors. For field scale, the recommended value of $C_{d}$ is $\sim 1$. This is based on the early measurement whereby $C_{d}=0.9$ was obtained by Forristall (1996) to fit the measured current blockage on the Bullwinkle platform in a high current associated with a Gulf of Mexico loop eddy.

\section{Conclusions}

This paper documents laboratory-scale experimental measurements on a scaled jacket model responding dynamically in a large towing tank when subjected to a range of focussed waves and embedded focussed wave in smaller regular wave background, all with steady current present (by towing the jacket using a carriage). The surface elevation time history next to the jacket model is measured for each test. For static tests where the jacket model is rigidly supported, the force through the force transducer (ground) is measured, hence the applied force is directly measured. For dynamic tests where the same jacket model is allowed to respond dynamically, the force through the springs (ground) as well as model displacement are both measured. The applied force is thus inferred using the transfer function derived from the equation of motion of a single degree-of-freedom 
oscillator. The resonant frequency of the jacket model is designed to be higher than the dominant wave frequency $(1.9 \times$ and $2.4 \times)$.

Numerical simulations are conducted in CFD using a porous tower model with distributed embedded Morison stresses representing both drag and inertia contributions to the total applied loads on the entire jacket structure. The Morison stresses vary in time according to the relative velocity formulation. Three methods are presented. In Method 1, it is necessary to solve for the modified flow field by internally coupling the CFD solver to a time-domain ODE solver to feedback the structural dynamics. Method 2 makes use of approximate expanded form of the Morison relative-velocity, which is much easier to implement. Method 3 is a checking procedure, using measured structural responses as input to the simulations. Good agreement among all three methods is achieved. More importantly, reasonably good agreement between numerical predictions and measurements is obtained, all using a single invariant set of Morison $C_{d}=1.3$ and $C_{m}=2.0$ for large range of flow structures with non-zero different current speeds. The generality of the results without the influence of Keulegen-Carpenter number wake-related effects is consistent with the previous results of Santo et al. (2018a). It is worth stressing that the numerical predictions generally slightly over-estimate the total force, and hence under-estimate the model displacement. Given the slight conservatism from the numerical results, we view this level of agreement as satisfactory.

Self consistency of the numerical model is shown by running the dynamic cases with a range of frequency ratios, with high frequency ratio resulting in the model response approaching that of the static case. It is also observed that the relative velocity formulation contributes to an additional damping into the dynamic system with an equivalent structural damping of $8 \%$ of critical. This is significantly larger than both the structural and assumed linear hydrodynamic damping combined (which is about $1 \%$ of critical) as quantified through free oscillation (push tests), and more than double the $2-3 \%$ of critical as recommended by industry standards for fixed structures (API RP $2 \mathrm{~A}, 2000)$. We also note that the SNAME standard for jack-ups apportions the damping to be $2 \%, 2 \%$, and $3 \%$ for structure, foundation and hydrodynamics, respectively (SNAME, 2008). In fact, very similar numerical predictions can be derived ignoring any structural damping. From the practical point of view, this is convenient because the estimation of the modelling of the damping term without incident far-field unsteadiness in the flow can now be avoided.

Overall, the agreement and consistency of the results demonstrates the validity of the Morison relative-velocity formulation, cast in terms of global wave-current-structure blockage for dynamically- 
responding structures in high frequency mode of vibration. For high frequency modes of vibration, Method 2 is recommended for practical industry adoption. Although low frequency mode of vibration was not considered in this paper, the structural velocity is also always smaller than the disturbed flow velocity, which renders the generality of Method 2 that spans across both high and low frequency mode of tower vibrations. To assess the dynamic response of a particular space-frame structure, one simply runs a static case in CFD using a porous block approach, and post-process the disturbed kinematics accordingly and feed these into a time-domain structural motion solver (such as the structural dynamics code USFOS) to extract the model displacement, and subsequently the applied hydrodynamic force.

\section{Appendix}

Figure 13 shows a comparison between the exact Morison relative-velocity term, i.e. $\left(u+u_{s}\right) \mid u+$ $u_{s}$ |, where $u$ is the combined wave and current velocity, and $u_{s}$ is the structural velocity, and the approximate expanded term, i.e. $u|u|+2 u_{s}|u|$, for a particular case where the ratio between current and structural velocity to wave velocity is $1 / 3$, respectively. The difference between the two terms is also shown as an error term. As apparent from the figure, the error term is relatively small, and changes sign within a wave cycle for a periodic oscillation. It seems unlikely that such a force error arising from the use of the approximate expanded term (Method 2) is important for any type of dynamically-responding structure.

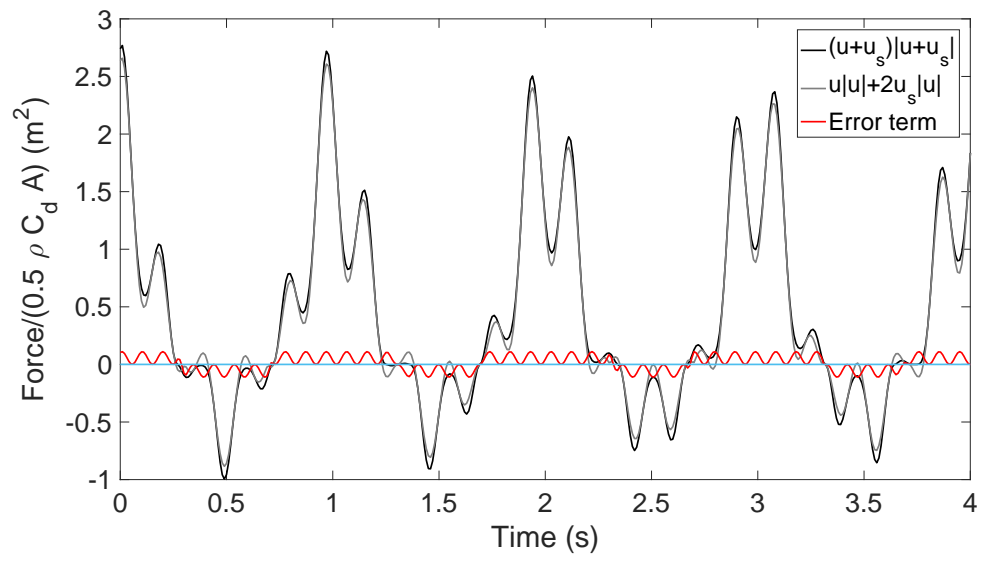

Figure 13: Comparison of the Morison relative-velocity term (black line) and the approximate expanded term (grey line) with the error term (red line) resulting from the difference between the two terms. 


\section{Acknowledgements}

We acknowledge the support from Singapore Maritime Institute (SMI) through grant number SMI-2015-AIMP-030. We would also like to acknowledge the use of the NUS High Performance Computing (HPC) facility in carrying out this work. The technicians at Kelvin Hydrodynamics Laboratory provided a high quality facility and technical service. We also thank Prof. Mike Efthymiou of the University of Western Australia for useful discussions.

\section{References}

API RP 2A, 2000. Recommended practice for planning, designing, and constructing fixed offshore platforms-working stress design. API RP2A-WSD 21st Edition with Erratas and Supplements 1, $130-132$.

Berberović, E., van Hinsberg, N. P., Jakirlić, S., Roisman, I. V., Tropea, C., 2009. Drop impact onto a liquid layer of finite thickness: dynamics of the cavity evolution. Physical Review E Statistical, Nonlinear, and Soft Matter Physics 79 (3), 036306.

Brackbill, J. U., Kothe, D. B., Zemach, C., 1992. A continuum method for modeling surface tension. Journal of Computational Physics 100 (2), 335-354.

Burrows, R., Tickell, R. G., Hames, D., Najafian, G., 1997. Morison wave force coefficients for application to random seas. Applied Ocean Research 19 (3-4), 183-199.

Das, S., Sahoo, T., Meylan, M. H., 2018. Dynamics of flexural gravity waves: from sea ice to Hawking radiation and analogue gravity. Proceedings of the Royal Society A: Mathematical, Physical and Engineering Sciences 474 (2209), 20170223.

Forristall, G. Z., 1996. Measurements of current blockage by the Bullwinkle platform. Journal of Atmospheric and Oceanic Technology 13 (6), 1247-1266.

Glauert, H., 1933. Wind tunnel interference on wings, bodies and airscrews. Tech. rep., Aeronautical Research Council Londong (UK).

Haritos, N., 2007. Introduction to the analysis and design of offshore structures-an overview. Electronic Journal of Structural Engineering 7 (Special Issue: Loading on Structures), 55-65. 
Jacobsen, N. G., Fuhrman, D. R., Fredsøe, J., 2012. A wave generation toolbox for the open-source CFD library: OpenFOAM ${ }^{\circledR}$. International Journal for Numerical Methods in Fluids 70 (9), 1073-1088.

Jonsson, I. G., 1990. Wave-current interactions. The Sea: Ocean Engineering Science 9, 65-119.

Lighthill, J., 1986. Fundamentals concerning wave loading on offshore structures. Journal of Fluid Mechanics 173, 667-681.

Maskell, E. C., 1963. A theory of the blockage effects on bluff bodies and stalled wings in a closed wind tunnel. Tech. rep., Aeronautical Research Council Londong (UK).

Merz, K. O., Moe, G., Gudmestad, O. T., 2009. A review of hydrodynamic effects on bottom-fixed offshore wind turbines. In: Proc. of the 28 th ASME International Conference on Ocean, Offshore and Arctic Engineering. pp. 927-941.

Moe, G., Verley, R. L. P., 1980. Hydrodynamic damping of offshore structures in waves and currents. In: Offshore Technology Conference, OTC 3798.

Morison, J. R., O’Brien, M. P., Johnson, J. W., Schaaf, S. A., 1950. The force exerted by surface waves on piles. Journal of Petroleum Technology 2 (5), 149-154.

Peregrine, D. H., 1976. Interaction of water waves and currents. In: Advances in Applied Mechanics. Vol. 16. Elsevier, pp. 9-117.

Santo, H., Stagonas, D., Buldakov, E., Taylor, P. H., 2017. Current blockage in sheared flow: Experiments and numerical modelling of regular waves and strongly sheared current through a space-frame structure. Journal of Fluids \& Structures 70, 374-389.

Santo, H., Taylor, P. H., Bai, W., Choo, Y. S., 2014a. Blockage effects in wave and current: 2D planar simulations of combined regular oscillations and steady flow through porous blocks. Ocean Engineering 88, 174-186.

Santo, H., Taylor, P. H., Bai, W., Choo, Y. S., 2015. Current blockage in a numerical wave tank: 3D simulations of regular waves and current through a porous tower. Computers \& Fluids 115, $256-269$ 
Santo, H., Taylor, P. H., Day, A. H., Nixon, E., Choo, Y. S., 2018a. Current blockage and extreme forces on a jacket model in focussed wave groups with current. Journal of Fluids and Structures $78 \mathrm{C}, 24-35$.

Santo, H., Taylor, P. H., Williamson, C. H. K., 2018b. On the Morison hydrodynamic forces on perforated flat plates in combined steady, low frequency and high frequency motion. Journal of Fluids and Structures (under review).

Santo, H., Taylor, P. H., Williamson, C. H. K., Choo, Y. S., 2014b. Current blockage experiments: force time histories on obstacle arrays in combined steady and oscillatory motion. Journal of Fluid Mechanics 739, 143-178.

Santo, H., Taylor, P. H., Williamson, C. H. K., Choo, Y. S., 2018c. The relative-velocity version of the morison equation for obstacle arrays in combined steady, low and high frequency motion. Journal of Fluid Mechanics 842, 188-214.

Shafiee-Far, M., Massie, W. W., Vugts, J. H., 1996. The validity of Morison equation extensions. In: Offshore Technology Conference, OTC 8064.

SNAME, 2008. Guidelines for site specific assessment of mobile jack-up units. Technical \& Research Bulletin 5-5A.

Steele, K., 1986. Performance of the Lena guyed tower. In: Offshore Technology Conference, OTC 5255 .

Sumer, B. M., Fredsøe, J., 2006. Hydrodynamics Around Cylindrical Structures. Advanced Series on Ocean Engineering. World Scientific Publishing.

Taylor, P. H., 1991. Current blockage: reduced forces on offshore space-frame structures. In: Offshore Technology Conference, OTC 6519.

Taylor, P. H., Santo, H., Choo, Y. S., 2013. Current blockage: reduced Morison forces on space frame structures with high hydrodynamic area, and in regular waves and current. Ocean Engineering $57,11-24$.

Williamson, C. H. K., 1985. In-line response of a cylinder in oscillatory flow. Applied Ocean Research $7(2), 97-106$. 